\title{
Cancer Status: Mocb and tPSA Prostate Cancer Markers
}

\section{Marian Surma}

Department of Physics, Optics Laboratory, Adam Mickiewicz University, Poznań, Poland.

Email: msur@amu.edu.pl

Received August 27 $7^{\text {th }}, 2012$; revised September $30^{\text {th }}, 2012$; accepted October $10^{\text {th }}, 2012$

\begin{abstract}
On the basis of the results of magneto-optical studies and their comparison with the outcome of medical tests magneto-optical MOCB and tPSA serum markers of cancer/recovered patient are presented. Status of the cancer serum donor is recognized as presence of the laevorotatory optical active molecules of ${ }^{(-)} \rho$ density in serum while status of the recovered donor is recognized as quantitative domination of the dextrorotatory ${ }^{(+)} \rho$ molecules in serum. These molecular information carriers ${ }^{(-)} \rho$ and ${ }^{(+)} \rho$ are recorded by the magneto-optical circular birefringence MOCB effect in $B^{2}$ magnetic field. The laevo/dextrorotatory carriers are enantiomers in the case of ${ }^{(-)} \rho={ }^{(+)} \rho$ relations fulfilled for any individual cancer and the same recovered patient. The positive predictive value PPV of MOCB results is $100 \%$.
\end{abstract}

Keywords: Serum; Molecular MOCB Marker; tPSA Marker

\section{Introduction}

The experimental MOCB technique [1] applied for serum and enantiomers $B^{2}$ chirality investigation of ovarian cancer serum and the neat chiral tartaric acid were firstly published since 1997 . The possibility of differentiation between the cancer and non-cancer states on the basis of the magneto-optical results has been tested [2]. The MOCB data collected suggest that the blood serum of cancer patient contains a stable bio-molecular structure. The $B^{2}$ effect in serum induced birefringence $\alpha\left(B^{2}\right)^{\exp }=$ $\left(\alpha^{+}-\alpha^{-}\right)$, where $\alpha^{+}$and $\alpha^{-}$denotes the $B^{2}$ induced optical activity of the dextro- and laevorotatory molecular carriers. The physical basis of the MOCB method is that the $\alpha^{+}$rotation is bearing by the dextrorotatory while the $\alpha^{-}$ by the leavorotatory carriers. This statement has been supported by the results $[1,2]$ obtained for patients clinically diagnosed by standard medical treatment and by analysis of their serum magneto-optical characteristics. The later introduce pure molecular physics to the cancer marker searching while the bio-chemical methods concerns mainly a proteins.

Magneto-optical circular birefringence (MOCB) measurements indicate extraordinary result for a cancer donor and healthy donor cases despite the cancer blood donor is after successful therapy and/or donors are a different non-cancer patients. Molecular carriers in serum standing for the the magnetic field induced $B^{2}$ circular birefringence are carrying information on the cancer/healthy donor status by the result of experimentally measured $\alpha^{+}$ and $\alpha^{-}$.

\section{Experiment}

The magneto-optical rotation $\alpha\left(B^{2}\right)$ induced by the $B^{2}$ field in the serum samples studied is described as: $\alpha\left(B^{2}\right)$ $=b^{\exp } B^{2} L$, where $b^{\exp }=\alpha\left(B^{2}\right)^{\exp } / 2 B^{2} L$ and $L$ is the light path in the serum.

The serum samples have been irradiated with an argon laser beam having wave length $\lambda=488 \mathrm{~nm}$, at $T \approx 295 \mathrm{~K}$ and the effective excitation volume of the serum was $V_{\text {eff }}$ $=15.7 \mathrm{~mm}^{3}$.

Intensity of the magnetic field [3] acting on the serum sample was $B=(15-30 \mathrm{~T})$.

The experimental result of $b^{\exp }=\alpha\left(B^{2}\right)^{\exp } /\left(2 B^{2} L\right)$ is a measure of serum magneto-optical birefringence expressed by the $\alpha\left(B^{2}\right)^{\exp }=\left(\alpha^{+}-\alpha^{-}\right)$.

Magneto-optical $b^{\exp }$ marker, quadratic magnetic field induced circular birefringence ${ }^{(-+)} \alpha\left(B^{2}\right)^{\text {exp }}$, effective natural optical activity $\alpha^{\text {exp }}$, density number ${ }^{(-)} \rho$ of the laevorotatory carriers, density number ${ }^{(+)} \rho$ of the dextrorotatory carriers are representative for the clinically diagnosed prostate cancer patient.

Magneto-optical characteristic of serum is defined by the $b^{\text {exp }}=b^{(-)}+b^{(+)}$markers [2]. The $b^{-}$marker is described by the relation $b^{\exp (-)} \rho=-4.114 \times 10^{28} S_{q}$ while serum magneto-optical result of $b^{+}$marker is described by the relation $b^{\exp \left({ }^{+}\right)} \rho=2.786 \times 10^{11} R_{q}$. where $S_{q}$ and $R_{q}$ denote the tensors: the electric quadrupolar and the magnetic dipolar optical polarizability of the laevorotatory and dextrorotatory molecules, respectively. The ${ }^{(-)} \rho$ denotes the density number of the laevo- and ${ }^{(+)} \rho$ of the dextrorotatory carriers in blood serum standing for the 
magnetic field induced circular birefringence of chiral media in $B^{2}$ magnetic field. Magneto-optical circular birefringence $(M O C B)$ measurements indicate extraordinary result for a cancer donor case: $b^{(-)} \neq 0, b^{\exp }<0$ and for healthy donor case: $b^{(+)} \neq 0, b^{\exp }>0$, despite the cancer blood donor is after successful therapy and/or donors are a different non-cancer patients.

\section{Results}

Table 1 gives a clear presentation of the prostate cancer diagnose and an outlook on the patient recovered status after the 45 Gy radiotherapy and 15 Gy brachytherapy processes. The laevorotatory molecular carriers quantitative MOCB representations are given by: $b^{\exp }<0,{ }^{(-)} \rho$ and $S_{q}$ data of a cancer status serum and by the superimposed dextrorotatory molecular carriers in serum of the same donor after successfully therapy: $b^{\exp }>0,{ }^{(+)} \rho$ and $R_{q}$ results. The $\alpha\left(B^{2}\right)^{\exp }, b^{\exp }$ and $\alpha^{\exp }$ experimental data of the cancer/healthy blood donor samples are within an experimental error of $+/-5 \%$.

\section{Discussion}

The MOCB prostate cancer marker $b^{(-)}$of patient's serum is carrying information on the density number of the magneto-optical active laevorotatory carriers ${ }^{(-)} \rho=-1.79$ $\times 10^{22} b^{(-)} \mathrm{mm}^{-3}$.

For the $b^{\text {exp }}= \pm 17 \times 10^{-5} \mathrm{deg} \cdot \mathrm{T}^{-2} \cdot \mathrm{mm}^{-1}$ the results of ${ }^{(-)} \rho$ and ${ }^{(+)} \rho$ are calculated (Table 1). This is a result of a pure physical analyze which introduce the molecular description of cancer status and prove coexistence of the cancer laevorotatory (-) and of the recovered dextrotrorotatory $(+)$ information carriers in serums. It seems, status of $(-)$ data $b^{\exp }=-17.0 \times 10^{-5} \mathrm{deg} \cdot \mathrm{T}^{-2} \cdot \mathrm{mm}^{-1},{ }^{(-)} \rho=3.04 \times 10^{18}$ carriers $/ \mathrm{mm}^{3}$ of the laevorotatory carriers, (cancer case), and of the $(+)$ data $b^{\text {exp }}=16.67 \times 10^{-5} \mathrm{deg} \cdot \mathrm{T}^{-2} \cdot \mathrm{mm}^{-1},{ }^{(+)} \rho$ $=2.98 \times 10^{18}$ carriers $/ \mathrm{mm}^{3}$ of the dextrorotatory carriers, (recovered patient case), are enantiomers. This statement is accepted (within $+/-5 \%$ ) by the MOCB experimental results of $-b^{(-)},{ }^{(-)} \rho, \alpha_{(-)}^{\exp }$ and $b^{(+)},{ }^{(+)} \rho, \alpha_{(+)}^{\exp }$.

These coherences are equivalent to the MOCB effect detected for a neat enantiomers [3] and the resultant findings of $\alpha_{(-)}^{\exp }=\alpha_{(+)}^{\exp }$ for cancer and recovered patient serums are very useful to support a remark: serum contain a lot of different proteins and molecular structures which are optical active and are forming a total optical activity $\alpha^{\exp }$ of the cancer case patient serum status ${ }^{(-)} \rho \gg$ ${ }^{(+)} \rho$. During a successfully therapy the density numbers of ${ }^{(-)} \rho$ and ${ }^{(+)} \rho$ carriers in serum are changing and finally the effective relation ${ }^{(-)} \rho \ll{ }^{(+)} \rho$, in serum of the recovering patient, is stabilized. That allowing to observe the dextrorotatory enantiomer in serum of the recovered patient status. Simply, the natural optical activity $\alpha^{\exp }$ of any serums contain: $\quad \alpha^{\text {exp }}={ }^{(-)} \rho\left\{-^{p . m .} \alpha(q)\right\}+{ }^{(+)} \rho\left\{{ }^{p . m .} \alpha(d)\right\}+\alpha_{t}$ of the $B^{2}$ induced effective birefringence of that medium. The $\alpha_{t}$ denote birefringence of the optical active proteins which are not bearing the ${ }^{(+)} \rho$ and ${ }^{(-)} \rho$ carriers while $\left\{{ }^{p . m .} \alpha(q)\right\}$ and $\left\{{ }^{p . m .} \alpha(d)\right\}$ are the electric quadrupolar, $\alpha(q)$, and the magnetic dipolar, $\alpha(d), B^{2}$ induced laevorotatory/dextrorotatory birefringence of a single $B^{2}$ active laevorotatory and dextrorotatory carriers, respectively.

Same carriers of the natural optical active structures in serum are laevorotatory electric quadrupole. Their density number of ${ }^{(-)} \rho$ dominate in serum of the cancer donor as in that medium ${ }^{(-)} \rho \gg{ }^{(+)} \rho$, however a density number ${ }^{(+)} \rho$ of the dextrorotatory magnetic dipole carriers are also present in the same serum.

The $\left\{{ }^{p . m .} \alpha(d)\right\}=-\left\{{ }^{p . m .} \alpha(q)\right\}$ relation, valid for enantiomers, gives: $\alpha_{(-)}^{\exp }=\alpha_{(+)}^{\exp }$.

Table 1. Clinically diagnosed patient ${ }^{*}$; radiotherapy $50 \mathrm{~Gy}^{* *}$; brachytherapy $15 \mathrm{~Gy}^{* * *}$.

\begin{tabular}{ccccccc}
\hline \multicolumn{7}{c}{ MOCB $/$ tPSA results: biopsy } \\
\hline $\mathrm{tPSA} /(\mathrm{ng} / \mathrm{mL})$ & $7.570^{*}$ & $6.100^{* *}$ & $2.270^{* * *}$ & 1.183 & 0.723 & 0.416 \\
$10^{5} b^{\mathrm{exp}} /\left(\mathrm{deg} \cdot \mathrm{T}^{-2} \cdot \mathrm{mm}^{-1}\right)$ & -17.0 & 12.03 & 18.39 & 16.67 & 9.36 & 12.34 \\
$10^{3} \alpha\left(B^{2}\right)^{\mathrm{exp}} /\left(\mathrm{deg} \cdot \mathrm{T}^{-2} \cdot \mathrm{mm}^{-3}\right)$ & -1.7 & 1.2 & 1.83 & 1.66 & 0.93 & 1.23 \\
$10^{3} \alpha^{\exp }(B=0) /\left(\mathrm{deg} \cdot \mathrm{mm}^{-3}\right)$ & 47.28 & 74.23 & 77.28 & 47.30 & 128.96 & 81.81 \\
$10^{-18(-)} \rho /\left(\mathrm{carriers} \cdot \mathrm{mm}^{-3}\right)$ & 3.04 & - & - & - & - & - \\
$10^{-18(+)} \rho /\left(\mathrm{carriers} \cdot \mathrm{mm}^{-3}\right)$ & - & - & - & 2.98 & - & - \\
$10^{51} S_{q} /\left(\mathrm{deg}{ }^{-1} \cdot \mathrm{T}^{-2} \cdot \mathrm{J}^{-1} \cdot \mathrm{A}^{2} \cdot \mathrm{m}^{3} \cdot \mathrm{s}^{2}\right)$ & 1.35 & - & - & - & - & - \\
$10^{34} R_{q} /\left(\mathrm{T}^{-2} \cdot \mathrm{J}^{-1} \cdot \mathrm{A}^{2} \cdot \mathrm{m}^{3} \cdot \mathrm{s}\right)$ & - & & & 2.05 & & \\
Enantiomers & $(-)$ & & & & & \\
\hline
\end{tabular}

Date of therapy result: Radio ${ }^{* *} /$ Brachy $^{* * *} ;(2009.05 .15)^{*} ;$ (2009.07.02) $^{* *} ;(2009.08 .17)^{* * *} ;(2011.02 .17),(2011.08 .31)$, (2012.02.28). 
On the other hand, a correct density number ${ }^{(-)} \rho$ of the cancer information carriers presence in serum acted on by $B^{2}$ magnetic field is out-coming from the relations:

$$
{ }^{(-)} \rho=-\alpha\left(B^{2}\right)^{\exp } /\left\{-{ }^{p \cdot m .} \alpha\left(B^{2}\right)\right\}
$$

where [4] $\left\{-^{p . m \cdot} \alpha\left(B^{2}\right)\right\}=-4114 \times 10^{28} S_{q}$ as from Equations (11) and (14) [2] the relation $4.114 \times 10^{28} S_{q}=$ $2.786 \times 10^{11} R_{q}$ is fulfilled by any equivalent serums of $(-/+)$ enantiomer status defined by $b^{(-)} f^{(-)} \rho=b^{(+)} \rho^{(+)} \rho$. The $(-)$ and (+) data (Table 1) gives: $S_{q}=1.35 \times 10^{-51}, R_{q}=$ $2.05 \times 10^{-34}$ and data of $\alpha_{t}=45.6 \times 10^{-3} \mathrm{deg} / \mathrm{mm}^{3}$ indicate presence of only $3.7 \%$ of the $B^{2}$ active quadrupolar electric carriers in the analyzed prostate cancer serum. Experimentally recorded effective MOCB birefringence $b^{\exp (-)} \rho=$ const, $S_{q}=$ const, (Equation (17)) [2], and results of the present paper introduce origin laevorotatory quadrupolar electric carrier marked the cancer marker $b^{\text {exp }}<0$.

\section{REFERENCES}

[1] M. Surma, "Magneto-Optical Circular Birefringence of a Chiral Medium in High Magnetic Field," Molecular Physics, Vol. 90, No. 6, 1997, pp. 993-997. doi: $10.1080 / 00268979709482683$

[2] M. Surma, "Magnetooptical Characterization of Human Blood Serum: Correlation between Neoplasmic Changes and Their Biomolecular Information Carriers," Physics and Chemistry of Liquids, Vol. 45, No. 3, 2007, pp. 271279. doi:10.1080/00319100600620912

[3] M. Surma, "Experimental Evidence of the $\mathrm{B}^{2}$ and $\mathrm{B}^{3}$ Dependent Circular Birefringence of Chiral Molecules in High Magnetic Fields," Molecular Physics, Vol. 93, No. 2, 1998, pp. 271-278. doi:10.1080/00268979809482210

[4] R. Zawodny, S. Woźniak and G. Wagnier'e, "On Quadratic dc Magnetic Field-Induced Circular Birefringence and Dichroism in Isotropic Chiral Media," Molecular Physics, Vol. 91, No. 91, 1997, pp. 165-172. 